Pacific Journal of Mathematics

FINITE PERMUTATION GROUPS WITH LARGE ABELIAN 


\title{
FINITE PERMUTATION GROUPS WITH LARGE ABELIAN QUOTIENTS
}

\section{G. Kovács and Cheryl E. Praeger}

\begin{abstract}
We show that if $G$ is a group of permutations on a set of $n$ points and if $\left|G / G^{\prime}\right|$ denotes the order of its largest abelian quotient, then either $\left|G / G^{\prime}\right|=1$ or there is a prime $p$ dividing $\left|G / G^{\prime}\right|$ such that $\left|G / G^{\prime}\right| \leq p^{n / p}$. Equality holds if and only if $G$ is a $p$-group which is the direct product of its transitive constituents, with each of those having order $p$, except when $p=2$ in which case one must also allow as transitive constituents the groups of order 4 , the dihedral group of order 8 and degree 4 , and the extraspecial group of order 32 and degree 8.
\end{abstract}

1. Introduction. In this paper we obtain upper bounds on the orders of abelian quotients of permutation groups in terms of the degrees of the groups, and identify the groups which attain these bounds. First we consider abelian $p$-quotients for a given prime $p$.

Recall that a constituent of a permutation group is the restriction of the group to some union of orbits, the restrictions to single orbits being the transitive constituents. By a transitive non- $p^{\prime}$-constituent we mean a transitive constituent whose order is divisible by $p$. The largest $p^{\prime}$-constituent is the restriction to the union of those orbits (if any) on which the group acts as a $p^{\prime}$-group.

THEOREM. If $G$ is a group of permutations on a finite set and if $k p$ denotes the number of points moved by a Sylow p-subgroup of $G$, then the largest abelian p-quotient of $G$ has order at most $p^{k}$. This maximum is achieved by $G$ if and only if $G$ is the direct product of its largest $p^{\prime}$-constituent (if any) and of its transitive non- $p^{\prime}$-constituents, each of the latter being from the following list of groups:

(i) $C_{p}$, of order and degree $p$;

(ii) $C_{4}, C_{2} \times C_{2}$, and $D_{8}$, of degree 4 , and the central product $D_{8} \curlyvee D_{8}$ of order 32 and degree 8 , when $p=2$;

(iii) the affine groups $\operatorname{AGL}(1,3)$ and $\operatorname{AGL}(1,5)$, when $p=2$;

(iv) the affine group $\mathrm{AGL}(1, p+1)$, when $p+1$ is a power of 2 . 
COROLlARY. If $G$ is a permutation group of degree $n$ and if $\left|G / G^{\prime}\right|$ denotes the order of its largest abelian quotient, then either $\left|G / G^{\prime}\right|=1$ or $\left|G / G^{\prime}\right|$ has a prime divisor $p$ such that $\left|G / G^{\prime}\right| \leq p^{n / p}$. Consequently, $\left|G / G^{\prime}\right| \leq 3^{n / 3}$. Given a prime $p$, one has $\left|G / G^{\prime}\right|=p^{n / p}$ if and only if $G$ is the direct product of its transitive constituents and each constituent is as in (i) or (ii) above (so in particular $G$ is a p-group).

The original motivation for this investigation came from a problem in [2] about the minimal (faithful) degree of an abstract group. Let $\mu(G)$ be the least integer for which $G$ has a faithful permutation representation of degree $\mu(G)$.

Conjecture. If $G / N$ is abelian, then $\mu(G / N) \leq \mu(G)$.

Our theorem implies that this holds whenever $G / N$ is an elementary abelian $p$-group. Indeed, in that case, if $|G / N|=p^{r}$ then $\mu(G / N)=$ $r p$. Consider $G$ a permutation group of degree $\mu(G)$ and denote by $k p$ the number of points moved by a Sylow $p$-subgroup: then obviously $k p \leq \mu(G)$. By the theorem we have $p^{r}=|G / N| \leq p^{k}$ so $r \leq k$, and hence $\mu(G / N)=r p \leq k p \leq \mu(G)$. Further, the theorem enables one to identify the groups $G$ and the elementary abelian $p$-quotients $G / N$ for which $\mu(G / N)=\mu(G)$. In general, the conjecture remains open.

A second motivation came from a question of $\mathrm{L}$. Babai, who asked (in a private communication) for an upper bound on $\left|G / G^{\prime}\right|$ for transitive $G$ of degree $n$. The bound provided by the corollary is exponential in $n$; is there a better one for transitive groups? We expect there is one which is exponential in $n(\log n)^{-1 / 2}$. For examples showing that one cannot hope for improvements beyond that, see Audu [1], Kovács and Newman [5].

Finally, we note that $\left|G / G^{\prime}\right| \leq p^{n / p}$ may hold for more than one, yet need not hold for all, prime divisors $p$ of $\left|G / G^{\prime}\right|$. For example, when $G$ is $C_{2} \times C_{3} \times C_{5}$ of degree $2+3+5$, one has

$$
3^{10 / 3}=9^{5 / 3}>8^{5 / 3}=2^{10 / 2}>\left|G / G^{\prime}\right|>5^{10 / 5} .
$$

2. Preliminaries. We shall need some elementary facts concerning (sub)direct products of (abstract) finite groups. For a fixed prime $p$ and each finite group $G$, write $G^{*}=G^{\prime} \mathbf{O}^{p}(G)$, so the largest abelian $p$-quotient of $G$ is $G / G^{*}$. Let $p^{\psi(G)}$ denote the maximum of the orders of the abelian $p$-sections of $G$ (that is, of the abelian $p$-quotients $K / L$ where $L \unlhd K \leq G)$. Let $G$ be a subdirect product of $A$ and $B$ : that is,

$$
G \leq A \times B=A G=B G .
$$


It is clear that for the mutual commutator subgroup $[A \cap G, A]$ we have

$$
[A \cap G, A] \leq A \cap G^{\prime},
$$

and that

$$
\text { if } A \leq G \text { then } G=A \times B \text {. }
$$

Obviously, $\psi(A)+\psi(B) \leq \psi(A \times B)$, and it is easy to see that in fact

$$
\psi(A)+\psi(B)=\psi(A \times B) .
$$

Indeed, let $G$ be a subgroup of $A \times B$ such that

$$
p^{\psi(A \times B)}=\left|G / G^{*}\right|:
$$

towards showing that $\left|G / G^{*}\right| \leq p^{\psi(A)+\psi(B)}$, we first replace $A$ and $B$ by $A \cap B G$ and by $A G \cap B$, respectively, so (1) holds, and then argue as follows. Note that

$$
\begin{gathered}
(A \cap G) G^{*} / G^{*} \cong(A \cap G) /\left(A \cap G^{*}\right), \\
G /(A \cap G) G^{*}=G /\left(G \cap A G^{*}\right) \cong A G / A G^{*}=A B / A G^{*} \cong B /\left(A G^{*} \cap B\right), \\
(A \cap G)^{*} \leq A \cap G^{*}, \\
(B \cap G)^{*} \leq B \cap G^{*}
\end{gathered}
$$

so (see Figure 1)

$$
\begin{aligned}
p^{\psi(A)+\psi(B)} & \leq p^{\psi(A \times B)}=\left|G / G^{*}\right| \\
& =\left|(A \cap G) G^{*} / G^{*}\right|\left|G /(A \cap G) G^{*}\right| \\
& =\left|(A \cap G) /\left(A \cap G^{*}\right)\right|\left|B /\left(A G^{*} \cap B\right)\right| \\
& \leq\left|(A \cap G) /(A \cap G)^{*}\right|\left|B / B^{*}\right| \\
& \leq p^{\psi(A)} p^{\psi(B)} .
\end{aligned}
$$
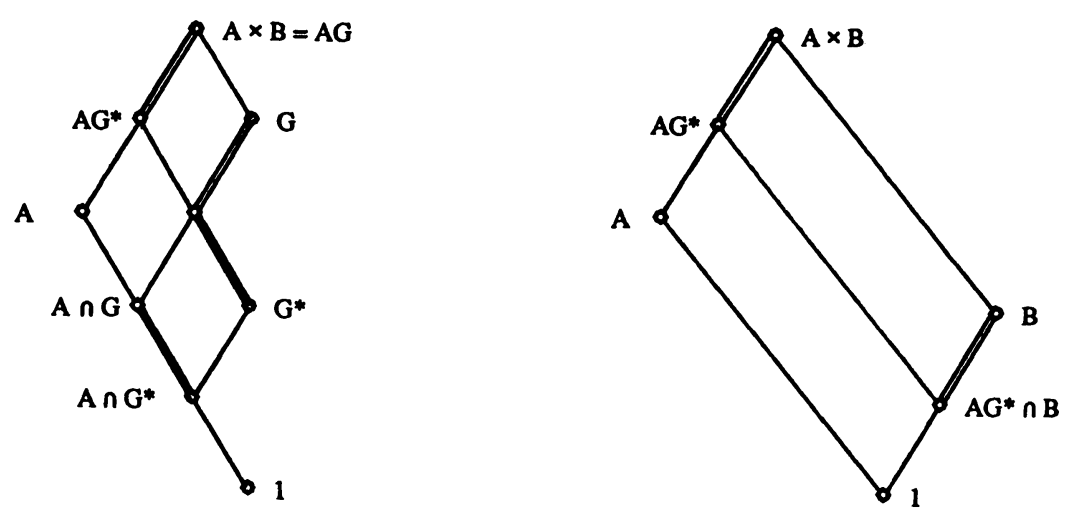

FIGURE 1 
It follows that all these inequalities are in fact equalities: so (4) is proved, and as a bonus we get that (1) and (5) together imply that

$$
\begin{aligned}
& (A \cap G)^{*}=A \cap G^{*}, \\
& \left|(A \cap G) /\left(A \cap G^{*}\right)\right|=p^{\psi(A)}, \\
& B^{*}=A G^{*} \cap B, \\
& \left|B / B^{*}\right|=p^{\psi(B)} .
\end{aligned}
$$

By symmetry, they also imply that

$$
\begin{aligned}
& (B \cap G)^{*}=B \cap G^{*}, \\
& \left|(B \cap G) /\left(B \cap G^{*}\right)\right|=p^{\psi(B)}, \\
& A^{*}=A \cap B G^{*}, \\
& \left|A / A^{*}\right|=p^{\psi(A)} .
\end{aligned}
$$

We shall also need some facts concerning permutation groups. The Sylow $p$-subgroup of the symmetric group of degree $p^{2}$ is sufficiently well known (the wreath product of two groups of order $p$ ):

(10) in this, no transitive subgroup has an abelian quotient of order $p^{p}$ except when $p=2$, in which case $C_{4}, C_{2} \times C_{2}$, and $D_{8}$ are the relevant examples.

In the holomorph of $D_{8}$, the left and the right translations form two copies of $D_{8}$ which centralize each other and meet precisely in their common centre; their product is the extraspecial group $D_{8} \curlyvee D_{8}$. In any holomorph the two translation groups have a common complement, namely the automorphism group: here that is abstractly isomorphic to $D_{8}$. The involution (in the symmetric group on the set of the elements of the original group) which inverts each group element always normalizes the automorphism group and interchanges the two translation groups; an order count shows that here the holomorph and that involution generate a Sylow 2-subgroup $P$ of the symmetric group of degree 8 (on the set of elements of the original $D_{8}$ ). We leave it to the reader to establish the following.

(11) The only transitive subgroup of $P$ which has an abelian quotient of order at least 16 is the product $D_{8} \curlyvee D_{8}$ of the two translation groups.

(12) Let $A$ and $A_{1}$ be groups of permutations on the same set, with $A_{1} \leq A$. Suppose that either $A$ is one of the transitive groups listed in the theorem or $A_{1}$ is one of the nilpotent groups listed there. If $\left[A_{1}, A\right] \leq A_{1}^{\prime}$ and, for the relevant prime, $\psi\left(A_{1}\right) \geq \psi(A)$, then $A_{1}=A$. 
(13) If $G$ is a transitive group of permutations on a set $\Omega$ and if the stabilizer $G_{\alpha}$ of a point $\alpha$, as group of permutations on $\Omega \backslash\{\alpha\}$, is one of the transitive groups listed in the theorem, then either $G$ is one of the affine groups listed there or $\left|G / G^{\prime}\right| \leq 2$ and a Sylow 2-subgroup of $G$ moves more than 2 points.

In verifying (13), it is convenient to distinguish two cases: under the hypotheses either $G_{\alpha}$ is nilpotent of class at most 2 so $G$ is soluble (Deskins, Janko, Thompson; see Huppert [3], IV.7.4), $\Omega$ is a vector space, and $G_{\alpha}$ acts on $\Omega$ faithfully as an irreducible linear group, or $G_{\alpha}$ is sharply 2-transitive, so $G$ is sharply 3 -transitive, in which case $\left|G / G^{\prime}\right| \leq 2$ (Zassenhaus; see Huppert and Blackburn [4], XI.2.1 and XI.2.6).

3. Proofs. First we prove the theorem for $p$-groups. In this case, it has the following simpler form.

If a $p$-group $G$ is a permutation group of degree $k p$, then $\left|G / G^{\prime}\right| \leq p^{k}$. Equality holds if and only if $G$ is the direct product of its transitive constituents and each of these is one of the groups listed in (i) and (ii) of the theorem.

In fact we shall show by induction on $k$ that if $\left|G / G^{\prime}\right| \geq p^{k}$ then $G$ is the product of its transitive constituents and each of these is one of the groups listed: $\left|G / G^{\prime}\right|=p^{k}$ is then automatic. The claim is obvious when $k=1$. For the inductive step, suppose $k>1$.

Consider first the case of intransitive $G$. Let $A$ be a nontrivial transitive constituent of $G$, and $B$ the restriction of $G$ to the union of the other orbits: then (1) holds. Say, the degree of $A$ is $l p$; then the degree of $B$ is $(k-l) p$, and the inductive hypothesis gives that $\psi(A) \leq l$, $\psi(B) \leq k-l$. By (4) we can now conclude that

$$
p^{\psi(A \times B)} \leq p^{k} \leq\left|G / G^{\prime}\right| \leq p^{\psi(A \times B)},
$$

so in fact $\psi(A)=l, \psi(B)=k-l,(5)$ holds and hence $(6)-\left(9^{\prime}\right)$ are also available. In particular, $\left|A / A^{\prime}\right|=p^{l}$ by $\left(9^{\prime}\right)$ and then the inductive hypothesis gives that $A$ is one of the listed groups. By (2), (7), and (12) with $A_{1}=A \cap G$, we have $A \cap G=A$ : so $G=A \times B$ by (3). As (9) gives $\left|B / B^{\prime}\right|=p^{k-l}$, the inductive hypothesis applied to $B$ completes this case of the inductive step.

Suppose next that $G$ is transitive. For this case (10) and (11) provide a more generous initial step: so we now assume not just $k>1$ but 
$k>p$ and $k p>8$. Let $H$ be a maximal subgroup of $G$ containing a point stabilizer: then $H$ is a normal subgroup of index $p$; it has $p$ orbits, which are permuted transitively by $G$, each having length $k$; of course now $k$ is a power of $p$ : set $k=m p$. Let $A$ be a transitive constituent of $H$ (the other transitive constituents being $G$-conjugates of $A$ ), and $B$ the restriction of $H$ to the union of the other orbits: then $H$ is a subdirect product of $A$ and $B$.

Consider the case $\left|(A \cap H) /\left(A \cap H^{\prime}\right)\right| \geq p^{m}$. From the inductive hypothesis we get that $\psi(A)=\psi(A \cap H)=m$; in particular $(A \cap H)^{\prime}=$ $A \cap H^{\prime}$ so by (2) we have

$$
[A \cap H, A] \leq(A \cap H)^{\prime} ;
$$

moreover $A \cap H$ is the direct product of its transitive constituents. As $A \cap H$ is normal in the transitive $A$, these constituents are all conjugate in $A$ : this contradicts $\left(2^{\prime}\right)$ unless $A \cap H$ is transitive. In that case $A \cap H$ is one of the listed groups, so $\left(2^{\prime}\right)$ and (12) with $A_{1}=A \cap H$ yields $A \cap H=A$, whence $H=A \times B$ by (3). Now $H$ is the product of the $G$-conjugates of $A$ which in the abelian $G / G^{\prime}$ have common image $A G^{\prime} / G^{\prime}$, hence $H / G^{\prime}=A G^{\prime} / G^{\prime}$ and so

$$
p^{m p} \leq\left|G / G^{\prime}\right|=p\left|H / G^{\prime}\right|=p\left|A /\left(A \cap G^{\prime}\right)\right| \leq p\left|A / A^{\prime}\right| \leq p^{m+1}
$$

which is impossible under our assumption that $m p=k>p$ and $k p>8$.

In the remaining case, that is, when $\left|(A \cap H) /\left(A \cap H^{\prime}\right)\right|<p^{m}$, we have that

$$
\left|H / H^{\prime}\right| \geq\left|H / G^{\prime}\right| \geq p^{m p-1}
$$

while Figure 1 (read with $H$ in place of $G$ and taking advantage of $\left.\mathbf{O}^{p}(H)=1\right)$ yields that

$$
\begin{aligned}
\left|H / H^{\prime}\right| & =\left|(A \cap H) H^{\prime} / H^{\prime}\right|\left|H /(A \cap H) H^{\prime}\right| \\
& =\left|(A \cap H) /\left(A \cap H^{\prime}\right)\right|\left|B /\left(A H^{\prime} \cap B\right)\right| .
\end{aligned}
$$

It follows that

$$
\left|B / B^{\prime}\right| \geq\left|B /\left(A H^{\prime} \cap B\right)\right|>p^{m p-1} / p^{m}, \quad \text { so }\left|B / B^{\prime}\right| \geq p^{m p-m} \text {. }
$$

As the degree of $B$ is $(m p-m) p$, the inductive hypothesis gives that $B$ is the direct product of its transitive constituents (which we know are $G$-conjugates of $A$ ) and each constituent is one of the groups listed under (i) and (ii). The only option consistent with $m p=k>p$ and $k p>8$ is $p=2, k=8, A \cong B \cong D_{8} \curlyvee D_{8}$. As $A \cap H$ and $B \cap H$ 
are disjoint conjugate subgroups of $G$, we must have $\left|G^{\prime}\right| \geq|B \cap H|=$ $|H| /|A|$; hence

$$
2^{8} \leq\left|G / G^{\prime}\right| \leq|G||A| /|H|=2|A|=2^{6} .
$$

This contradiction completes the inductive step, so (14) is proved.

Proof of the Theorem. We shall use induction, firstly on the degree of $G$ and secondly on the order of $G$. Accordingly, the inductive hypothesis will consist of two parts: the theorem is true for all groups whose degree is smaller than that of $G$, and also for all proper subgroups of $G$. As the theorem is a tautology when $G$ is a $p^{\prime}$-group and is valid by (14) whenever $G$ is a $p$-group, the initial step presents no problem. Indeed we can assume that $G$ is neither a $p$-group nor a $p^{\prime}$-group.

The first sentence of the theorem is an immediate consequence of applying the inductive hypothesis to a Sylow $p$-subgroup of $G$. The "if" part of the second sentence is obvious. To deal with the "only if" part, suppose that $\left|G / G^{\prime} \mathbf{O}^{p}(G)\right|=p^{k}$ : the task is to prove that $G$ has the required structure. Note that, since a Sylow $p$-subgroup of a subgroup of $G$ cannot move more than $k p$ points, by the inductive hypothesis we have that

a proper subgroup which supplements $G^{\prime} \mathbf{O}^{p}(G)$ in $G$ must have the required structure and its Sylow $p$-subgroup must move precisely $k p$ points.

The case of intransitive $G$ can be handled by adapting the corresponding step from the proof of (14): the relevant preliminaries in $\S 2$ had been prepared with this in mind. The only extra point is that now a transitive non- $p^{\prime}$-constituent should be chosen as $A$.

The next case we consider is that of a transitive $G$ whose degree is divisible by $p$. Then a point stabilizer cannot contain any Sylow $p$-subgroup of $G$; equivalently, a Sylow $p$-subgroup $P$ of $G$ can fix no point: so the degree of $G$ is $k p$. It follows that all proper supplements of $G^{\prime} \mathbf{O}^{p}(G)$ in $G$ are $p$-groups: for, by (15), they must have the required structure but now can have no affine constituents. Let $q$ be another prime divisor of $|G|$; by the Frattini argument, the normalizer $\mathbf{N}_{G}(Q)$ of a Sylow $q$-subgroup $Q$ supplements $G^{\prime} \mathbf{O}^{p}(G)$; by the previous sentence, $\mathbf{N}_{G}(Q)$ cannot be a proper subgroup: so $Q$ is normal in $G$. By a similar argument, $P Q=G$. As $Q$ is normal, its orbits form a system of imprimitivity; in particular, they have a common length, say $q^{b}$. The degree $k p$ being divisible both by $q^{b}$ and by $p$, neither $P$ 
nor $Q$ is transitive. Let $\Gamma$ and $\Gamma^{\prime}$ be distinct $P$-orbits and $\Delta$ a $Q$-orbit. Since $P Q=G$, each coset of $P$ meets each coset of $Q$ and so each $P$-orbit meets each $Q$-orbit: consequently $\Delta \nsupseteq \Gamma$ and we can choose a $\delta$ in $\Gamma^{\prime} \cap \Delta$. Since $P$ is the direct product of its transitive constituents (by (15)), the point stabilizer $P_{\delta}$ is transitive on $\Gamma$. Now we use that $\Delta$ is a block of imprimitivity for $G$ : it must be fixed (setwise) by $P_{\delta}$ because $\delta \in \Delta$, and cannot be fixed by $P_{\delta}$ because it meets but does not contain the $P_{\delta}$-orbit $\Gamma$. This contradiction shows that the case under consideration cannot arise.

It remains to consider transitive $G$ of degree prime to $p$. Our first aim is to show that in this case $G$ is primitive. Take any point stabilizer $G_{\alpha}$, any Sylow $p$-subgroup $P$ of $G$ contained in $G_{\alpha}$, and any maximal subgroup $M$ containing $G_{\alpha}$. Assume that $G_{\alpha}<M$ : we shall show that this leads to a contradiction. Consider the corresponding system of imprimitivity, and let $m$ denote the common length $\left|G_{\alpha}: M\right|$ of its blocks: note that $p$ does not divide $m$, so no $P$-orbit $\Gamma$ can be a union of blocks. If a block $\Delta$ meets two $P$-orbits $\Gamma, \Gamma^{\prime}$ without containing both of them, we get a contradiction as at the end of the previous paragraph. If a block $\Delta^{\prime}$ meets only one $P$-orbit $\Gamma$, then of course $\Delta^{\prime} \subset \Gamma$ so $\Gamma$ must also meet some block $\Delta$ which it does not contain; in turn, that $\Delta$ must meet another $P$-orbit $\Gamma^{\prime}$ as well, and the previous sentence applies. In the remaining case each block is a union of $P$ orbits, that is, $P$ fixes each block setwise: $P$ lies in the intersection, say $N$, of the conjugates of $M$. Note that $N \mathbf{O}^{p}(G)=G$. Since $N$ is normal in the transitive $G$, the transitive constituents of $N$ are all $G$-conjugate: in particular they are all isomorphic so none of them can be a $p^{\prime}$ group, and hence $N$ is their direct product by (15). As $G_{\alpha} N \leq M<G$, there is more than one transitive constituent; thus if $A$ is any one of them, a Sylow $p$-subgroup of $A$ cannot move as many as $k p$ elements, and the inductive hypothesis gives that $\left|A / A^{\prime} \mathbf{O}^{p}(A)\right|<p^{k}$. On the other hand, $N$ being the product of the conjugates of $A$ implies that $A G^{\prime} / G^{\prime}=N G^{\prime} / G^{\prime}$ whence $A G^{\prime} \mathbf{O}^{p}(G)=N G^{\prime} \mathbf{O}^{p}(G)=G$, and then

$$
\begin{aligned}
p^{k} & >\left|A / A^{\prime} \mathbf{O}^{p}(A)\right| \geq\left|A /\left(A \cap G^{\prime} \mathbf{O}^{p}(G)\right)\right| \\
& =\left|G / G^{\prime} \mathbf{O}^{p}(G)\right|=p^{k}
\end{aligned}
$$

is the desired contradiction.

This has proved that $G$ is primitive. The next aim is to show that $G$ is 2-transitive. By 18.4 in Wielandt's [6], no transitive constituent of $G_{\alpha}$ except that on $\{\alpha\}$ can be a $p^{\prime}$-group, so by (15) we know that $G_{\alpha}$ is the direct product of its transitive constituents. Let $\Gamma$ be a $G_{\alpha}$-orbit, 
other than $\{\alpha\}$, of shortest possible length, say, $d$. Choose a point $\gamma$ in $\Gamma$ and an element $h$ in $G$ such that $\alpha h=\gamma$; let $\Delta$ be the $G_{\gamma}$-orbit $\Gamma h$, and $\Delta^{\prime}$ the $G_{\gamma}$-orbit paired with $\Delta$ : that is, $\Delta^{\prime}$ consists of all the $\gamma g$ with $g \in\{g \in G \mid \gamma \in \Delta g\}$. By 16.3 in [6], the length of $\Delta^{\prime}$ is also $d$, while $\alpha \in \Delta^{\prime}$ because $\alpha=\gamma h^{-1}$ and $\gamma \in \Gamma=(\Gamma h) h^{-1}=\Delta h^{-1}$. Since $G_{\alpha}$ is the direct product of its transitive constituents, $G_{\alpha \gamma}$ acts transitively on each $G_{\alpha}$-orbit other than $\Gamma$ : hence all the $G_{\alpha \gamma}$-orbits of length less than $d$ lie in $\{\alpha\} \cup \Gamma$. On the other hand, $\{\gamma\} \cup \Delta^{\prime}$ is a union of $G_{\gamma}$-orbits; as this set has $d+1$ elements of which two, $\alpha$ and $\gamma$, are fixed by $G_{\alpha \gamma}$, it is a union of $G_{\alpha \gamma}$-orbits each of length less than $d$. Consequently $\{\gamma\} \cup \Delta^{\prime} \subseteq\{\alpha\} \cup \Gamma$; as both sets have cardinality $d+1$, they are in fact equal. It follows that this set is fixed (setwise) by $G_{\alpha}$ and by $G_{\gamma}$, so it is fixed by $G$ and is therefore the whole set, say $\Omega$, on which $G$ acts. In particular, $G_{\alpha}$ is transitive on $\Omega \backslash\{\alpha\}$.

By (15) it follows that $G_{\alpha}$ is one of the transitive groups listed, so (13) is applicable, and the proof of the theorem is complete.

Proof of the Corollary. Since the function $x^{1 / x}$ is decreasing when $x \geq e$,

$$
3^{1 / 3}>4^{1 / 4}=2^{1 / 2}>5^{1 / 5}>\cdots
$$

and so only the first sentence of the corollary needs additional justification. Suppose that is false. Let $n$ be the least integer for which counterexamples of degree $n$ exist, and let $G$ be a degree $n$ counterexample of minimal order. For a prime $p$ dividing $|G|$, let $P$ be a Sylow $p$-subgroup of $G$. By the Frattini argument applied with the normal subgroup $P G^{\prime}$, we have that $\mathrm{N}_{G}(P) G^{\prime}=G$ so $G / G^{\prime}$ is a homomorphic image of $\mathbf{N}_{G}(P) / \mathbf{N}_{G}(P)^{\prime}$ : as $|G|$ is minimal, this can only happen if $\mathbf{N}_{G}(P)=G$. Thus all Sylow subgroups of $G$ are normal: $G$ is nilpotent. By the theorem $G \neq P$, so $G=P \times Q$ with a nontrivial subgroup $Q$. Of course, $G / G^{\prime} \cong\left(P / P^{\prime}\right) \times\left(Q / Q^{\prime}\right)$. Any orbit of a direct product of a $p$-group $P$ and a $p^{\prime}$-group $Q$ may be viewed as the cartesian product $\Gamma \times \Delta$ of a $P$-orbit $\Gamma$ and a $Q$-orbit $\Delta$. If there were a $G$-orbit $\Gamma \times \Delta$ on which both $P$ and $Q$ acted nontrivially, one could replace it by the disjoint union $\Gamma \cup \Delta$, obtaining a smaller set on which $G$ still acted faithfully, and this would contradict the minimality of $n$. Thus if $a$ denotes the number of points moved by $P$ and $b$ the number moved by $Q$, we have

$$
a+b=n \text {. }
$$

By the minimality of $n$ we have $\left|P / P^{\prime}\right| \leq p^{a / p}$ and $\left|Q / Q^{\prime}\right| \leq q^{b / q}$ for some prime divisor $q$ of $\left|Q / Q^{\prime}\right|$ : then $\left|G / G^{\prime}\right| \leq p^{a / p} q^{b / q}$. Finally, by 
the last two displayed lines, $p^{a / p} q^{b / q}$ cannot be larger than both of $p^{n / p}$ and $q^{n / q}$, so $G$ is not a counterexample after all.

\section{REFERENCES}

[1] Muhammed Salihu Audu, Transitive permutation groups of prime-power order, D. Phil. thesis, Oxford, 1983.

[2] D. Easdown and C. E. Praeger, On the minimal faithful degree of a finite group, University of Western Australia Research Report, 1987.

[3] B. Huppert, Endliche Gruppen I, Springer-Verlag, Berlin Heidelberg New York, 1967.

[4] B. Huppert and N. Blackburn, Finite Groups III, Springer-Verlag, Berlin Heidelberg New York, 1982.

[5] L. G. Kovács and M. F. Newman, Generating transitive permutation groups, Quarterly J. Math. Oxford, (2) 39 (1988), to appear.

[6] Helmut Wielandt, Finite Permutation Groups, Academic Press, New York and London, 1964.

Received November 13, 1987.

AUSTRALIAN National University

CANBerRa, ACT 2601

AND

University of Western Australia

NedLANDS, WA 6009 


\section{PACIFIC JOURNAL OF MATHEMATICS \\ EDITORS}

\author{
V. S. VARADARAJAN \\ (Managing Editor) \\ University of California \\ Los Angeles, CA 90024 \\ HERBERT ClEMENS \\ University of Utah \\ Salt Lake City, UT 84112 \\ THOMAS ENRIGHT \\ University of California, San Diego \\ La Jolla, CA 92093
}

R. FINN

Stanford University

Stanford, CA 94305

HERMANN FLASCHKA

University of Arizona

Tucson, AZ 85721

VAUGhan F. R. JONES

University of California

Berkeley, CA 94720

STEVEN KERCKHOFF

Stanford University

Stanford, CA 94305

\section{ROBION KIRBY}

University of California

Berkeley, CA 94720

C. C. MOORE

University of California

Berkeley, CA 94720

HAROLD STARK

University of California, San Diego

La Jolla, CA 92093

\section{ASSOCIATE EDITORS}
R. ARENS
E. F. BECKENBACH
B. H. NEUMANN
F. WOLF
K. YOSHIDA

(1906-1982)

\section{SUPPORTING INSTITUTIONS}

UNIVERSITY OF ARIZONA

UNIVERSITY OF BRITISH COLUMBIA

CALIFORNIA INSTITUTE OF TECHNOLOGY

UNIVERSITY OF CALIFORNIA

MONTANA STATE UNIVERSITY

UNIVERSITY OF NEVADA, RENO

NEW MEXICO STATE UNIVERSITY

OREGON STATE UNIVERSITY

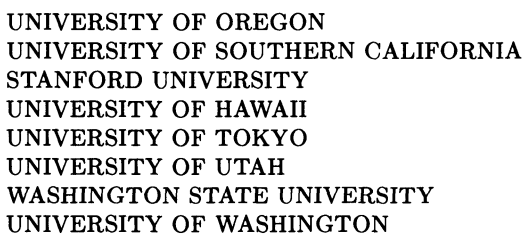

The Supporting Institutions listed above contribute to the cost of publication of this Journal, but they are not owners or publishers and have no responsibility for its content or policies.

\footnotetext{
Mathematical papers intended for publication in the Pacific Journal of Mathematics should be in typed form or offset-reproduced (not dittoed), double spaced with large margins. Please do not use built up fractions in the text of the manuscript. However, you may use them in the displayed equations. Underline Greek letters in red, German in green, and script in blue. The first paragraph must be capable of being used separately as a synopsis of the entire paper. In particular it should contain no bibliographic references. Please propose a heading for the odd numbered pages of less than 35 characters. Manuscripts, in triplicate, may be sent to any one of the editors. Please classify according to the scheme of Math. Reviews, Index to Vol. 39. Supply name and address of author to whom proofs should be sent. All other communications should be addressed to the managing editor, or Elaine Barth, University of California, Los Angeles, California 90024.

There are page-charges associated with articles appearing in the Pacific Journal of Mathematics. These charges are expected to be paid by the author's University, Government Agency or Company. If the author or authors do not have access to such Institutional support these charges are waived. Single authors will receive 50 free reprints; joint authors will receive a total of 100 free reprints. Additional copies may be obtained at cost in multiples of 50 .
}

The Pacific Journal of Mathematics is issued monthly as of January 1966. Regular subscription rate: $\$ 190.00$ a year (5 Vols., 10 issues). Special rate: $\$ 95.00$ a year to individual members of supporting institutions.

Subscriptions, orders for numbers issued in the last three calendar years, and changes of address should be sent to Pacific Journal of Mathematics, P.O. Box 969, Carmel Valley, CA 93924, U.S.A. Old back numbers obtainable from Kraus Periodicals Co., Route 100, Millwood, NY 10546.

The Pacific Journal of Mathematics at P.O. Box 969, Carmel Valley, CA 93924 (ISSN 0030-8730) publishes 5 volumes per year. Application to mail at Second-class postage rates is pending at Carmel Valley, California, and additional mailing offices. Postmaster: send address changes to Pacific Journal of Mathematics, P.O. Box 969, Carmel Valley, CA 93924.

PUBLISHED BY PACIFIC JOURNAL OF MATHEMATICS, A NON-PROFIT CORPORATION Copyright (C) 1989 by Pacific Journal of Mathematics 


\section{Pacific Journal of Mathematics}

\section{Vol. 136, No. 2 December, 1989}

A. K. Agarwal and David Bressoud, Lattice paths and multiple basic hypergeometric series ................................. 209

Adalberto Panobianco Bergamasco and Hermano de Souza Ribeiro, Uniqueness in a doubly characteristic Cauchy problem ........... 229

Thomas Curtis Craven and George Leslie Csordas, Jensen polynomials

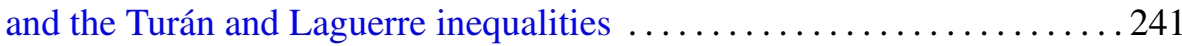

Gary R. Jensen and Marco Rigoli, Harmonic Gauss maps ............ 261

L. G. Kovács and Cheryl Elisabeth Praeger, Finite permutation groups with large abelian quotients $\ldots \ldots \ldots \ldots \ldots \ldots \ldots \ldots \ldots \ldots \ldots \ldots . \ldots \ldots$

Ken-ichi Maruyama, Localization of a certain subgroup of self-homotopy

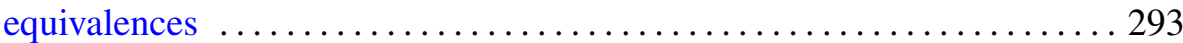

Tomasz Mazur, Canonical isometry on weighted Bergman spaces ......... 303

Bernt Karsten Oksendal, A stochastic Fatou theorem for quasiregular

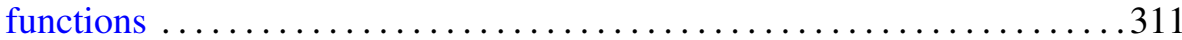

Ian Fraser Putnam, The $C^{*}$-algebras associated with minimal homeomorphisms of the Cantor set ........................ 329

Tom Joseph Taylor, Some aspects of differential geometry associated with

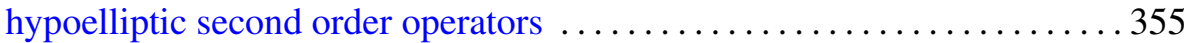

Tom Joseph Taylor, Off diagonal asymptotics of hypoelliptic diffusion equations and singular Riemannian geometry 